\title{
FIELD EVIDENCE OF THE EFFECTS OF PRO-SOCIALITY AND TRANSPARENCY ON COVID-19 APP ATTRACTIVENESS
}

\author{
Samuel Dooley \\ University of Maryland \\ sdooley1@cs.umd.edu
}

\author{
Dana Turjeman \\ University of Michigan \\ turji@umich.edu \\ Elissa M. Redmiles \\ Max Planck Institute for Software Systems \\ eredmiles@mpi-sws.org
}

\author{
John P. Dickerson \\ University of Maryland \\ john@cs.umd.edu
}

July 22,2021

\begin{abstract}
COVID-19 exposure-notification apps have struggled to gain adoption. Existing literature posits as potential causes of this low adoption: privacy concerns, insufficient data transparency, and the type of appeal used to pitch the pro-social behavior of installing the app. In a field experiment, we advertised CovidDefense, Louisiana's COVID-19 exposure-notification app, at the time it was released. We find that all three hypothesized factors - privacy, data transparency, and appeals framing - relate to app adoption, even when controlling for age, gender, and community density. Specifically, we find that collective-good appeals are effective in fostering pro-social COVID-19 app behavior in the field. Our results empirically support existing policy guidance on the use of collective-good appeals and offer real-world evidence in the on-going debate on the efficacy of such appeals. Further, we offer nuanced findings regarding the efficacy of transparency - about both privacy and data collection - in encouraging health technology adoption and pro-social COVID-19 behavior. Our results may aid in fostering pro-social public-health-related behavior and for the broader debate regarding privacy and data transparency in digital healthcare.
\end{abstract}

To combat SARS-CoV-2 - also known as "coronavirus" - and its associated illness COVID-19, countries and other entities have worked to develop vaccines and a variety of other mitigation tools. One such tool is contact-tracing technology that serves as the foundation for exposure-notification apps (COVID-19 apps) that can alert users when they have been exposed to coronavirus. These apps have been developed and deployed in 77 countries and U.S. states 1

Similar to other pro-social COVID-19 behaviors such as vaccination and mask adoption, greater adoption of COVID-19 apps improves their efficacy. Yet, adoption has been low, with the highest adoption rates per jurisdiction hovering around $30 \%$ and typical adoption rates closer to $10 \% 2^{2}$

Existing policy guidance [1] for COVID-19, which draws on prior work in health communication, encourages the use of tailored messaging to foster pro-social public health behavior such as the adoption of COVID-19 apps. We collaborated with the state of Louisiana to test the impact of tailored messaging on adoption of the state's COVID-19 exposure-notification app, CovidDefense, when the app was launched. Specifically, we test the impact of advertisements that contain two types of messaging recommended in existing guidance on encouraging pro-social COVID-19 behavior: (a) collective-good appeals and (b) transparency.

Collective-good appeals (i.e., pro-social messages that speak to community benefit) are suggested as a best practice by existing policy guidance [1]. However, the efficacy of such appeals is empirically debated [2] on the basis of

\footnotetext{
${ }^{1}$ See the Linux Public Health Foundation dashboard (https://landscape. Ifph.io/) for a running list of deployed COVID-19 apps.

${ }^{2}$ There has been little official reporting of COVID-19 app adoption rates outside of the popular press; we refer to https: //time.com/5905772/covid-19-contact-tracing-apps/for these adoption statistics.
} 
evidence from self-report data, laboratory experiments, and hybrid self-report tracking [3, 4]. Most closely related to our work, Munzert et al. tested the effect of presenting collective-good appeals in combination with privacy and functionality-related information in a video intervention to participants in an opt-in survey panel in Germany on the COVID-19 app adoption behavior of participants whose digital behavior could be tracked by the survey panel [4]. Their experiment found a null result, though this might be an artifact of some experimental limitation $3^{3}$ In contrast, our work isolates and focuses specifically on the impact of appeals in tailored messaging, presenting the first, to our knowledge, direct field evaluation in the general population of the efficacy of collective-good appeals in encouraging pro-social COVID-19 behavior. Specifically, we tested this in tailored advertising messaging used to encourage adoption of an exposure notification app at the time it was released to the population 4 Importantly, our work does not rely on surveys, online studies or an opt-in sample. Instead, we directly measure the outcome of interest: whether a prospective user clicks to download the app.

Existing policy guidance also encourages transparency in messaging. Prior self-report studies of COVID-19 exposure notification apps suggest that concerns regarding both privacy and data collection significantly relate to people's adoption intentions [7]. However, while prior research in the privacy domain (e.g., 8, 9) has found that increased transparency and sense of control regarding existing privacy and data collection may reduce concerns and increase willingness to share data, it is an open question whether such transparency can be effectively provided through tailored messaging [10].

To test the effects of collective-good appeals and transparency in messaging designed to encourage adoption of a COVID-19 app, we conducted our randomized field experiment on the Google Ads Platform using 14 different ads. Ads were randomly displayed to Louisiana residents and generated 7,010,271 impressions 5 The outcome measured was whether the user clicked the respective ad; those who clicked were redirected to the Louisiana Department of Public Health app download page (http://coviddefensela.com/) ${ }^{6}$

The results of our field study show that tailored messaging can effectively influence the pro-social behavior of installing a COVID-19 app. We find that significantly more people click on messages that use collective-good appeals than those that use individual-good appeals. Furthermore, in a series of moderation analyses, we find that transparency about data collection and privacy moderate this effect. Such differences suggest that digital privacy and data transparency can be effectively provided through tailored messaging. Moreover, our results shed light on how priming with an individual-good appeal increases gender and age differences in receptiveness to the ads and to the different privacy controls presented.

\section{Experimental Design}

Our experiment addresses three research questions:

RQ1: Is messaging that presents the benefit of app installation as a collective-good appeal (i.e., with societal benefit) more effective than messaging that appeals to individual-good?

RQ2: Is messaging that makes privacy transparent more effective than messaging that does not? And, which privacy transparency statements are most/least effective, those that: (a) broadly reassure people about privacy concerns, or those that specifically focus on enhanced control over data collection - through a statement emphasizing either (b) broad, non-technical privacy control or (c) technically concrete privacy control?

RQ3: Is messaging that makes data collection transparent (i.e., stating clearly what data is being collected) by the app more effective than messaging that does not inform potential users what data will be collected?

\footnotetext{
${ }^{3}$ Multiple limitations in the design of Munzert et al., as concluded in [5], include 1. the nature of the intervention, which involved exposure to a training video during a survey-based study rather than as part of real-world installation behavior and which combined multiple experimental messages, preventing isolation of the impact of the collective-good appeal from the other experimental factors, 2. the sample size, and 3. the opt-in nature of the participant pool.

${ }^{4}$ Banker and Park conducted a field study on the impact of collective-good appeals on clicks to CDC guidelines at the very beginning of the pandemic [6]. However, health information consumption and pro-social health behavior are importantly different constructs.

${ }^{5}$ As is typical in digital marketing campaigns, ads may be displayed during Google search more than once to the same user/IP address and thus the number of impressions is larger than the population of Louisiana.

${ }^{6} \mathrm{~A}$ user may have seen more than one ad because Google does not allow us to control this. However, if the user clicked on an ad, the click was associated with the specific ad on which they clicked.
} 


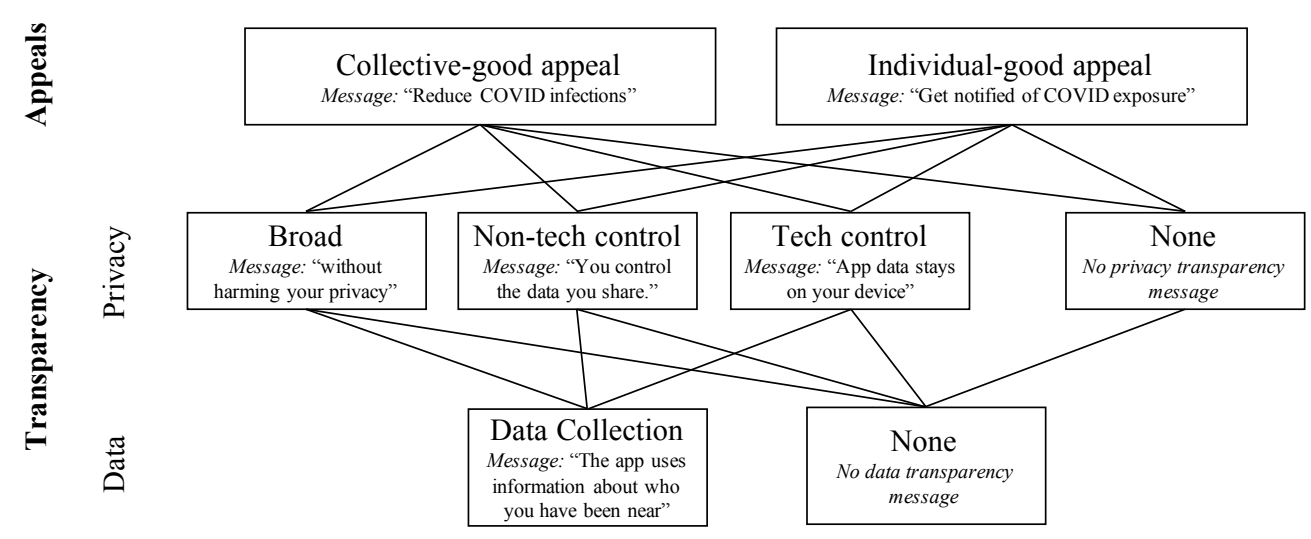

Figure 1: Experimental design for the 14 messages shown in the field study.

\footnotetext{
${ }^{7}$ Our study was approved by the University of Saarland ERB (20-09-3) and exempted from review by the Louisiana Department of Health IRB and the University of Michigan IRB.

${ }^{8}$ Prior work has validated the accuracy of this state-level targeting [11].

${ }^{9}$ The accuracy of these inferences has been validated against gold-standard social scientific probabilistic survey panels and other self-report data sources [12].
} 


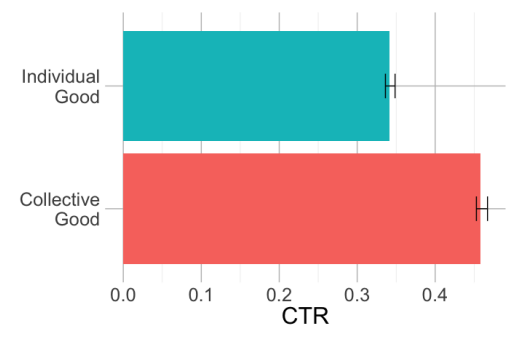

(a)

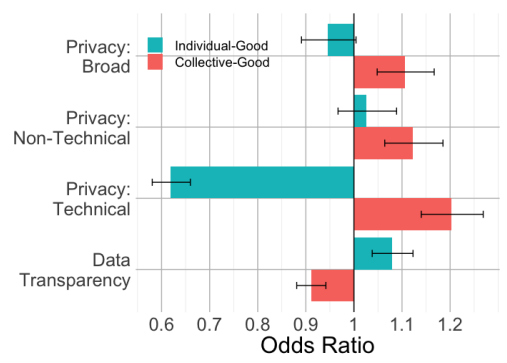

(b)

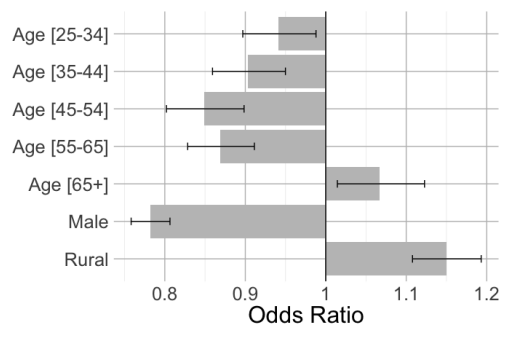

(c)

Figure 2: Main results from regression models. Error bars of 95\% confidence are given. (a) Overall CTRs for individualand collective-good appeals. (b) Odds ratio for each transparency statement modeled twice for ads presented with each of the two appeals. The individual-good appeal (in red) is above the collective-good appeal (in blue). Regression tables are Appendix Tables 4 and 5 . (c) Overall odds ratio for each demographic and geographic variable. Regression table is Appendix Table 6

The type of appeal (collective or individual) moderates the impact of the transparency statements. We conclude this by observing significant interactions between the appeal and the transparency statements in logistic regression models (Appendix Table 3). Subsequently, performing a regression on each appeal individually, we report the odds ratios and errors for the transparency statements for each appeal in Figure $2 \mathrm{~b}$

Messages with a collective-good appeal have a higher CTR when they have additional privacy transparency statements all three of the statements have O.R.s that range from 1.106 to 1.203 , all with $p<0.001$ - but result in lower CTR responses when paired with a data transparency statement, i.e., when ads explicitly mentions what data is being collected (O.R. $=0.911, p<0.001$; regression tables in Appendix Tables 4 and 5). On the other hand, messages with an individual-good appeal have a lower CTR when paired with a technical privacy transparency statement $(\mathrm{O} . \mathrm{R} .=0.619$, $p<0.001)$. However, the broad and non-technical control privacy transparency statements cannot be deemed to affect the CTR of the same individual-good appeal ( $p=0.070$ and $p=0.396$, respectively). Moreover, contrary to the negative effect of data transparency on CTR in the collective-good appeal condition, when data collection is made transparent in the individual-good appeal condition, we observe a higher CTR than in messages without such data transparency (O.R. $=1.08, p<0.001)$. We observe that, in a single regression model containing interactions between the transparency statements and the appeals, a data transparency statement reduces the difference in CTR between messages with collective- vs. individual-good appeals, while inclusion of a privacy statement increases the difference in CTR between messages with the two different appeals.

Thus, our findings regarding transparency are nuanced. Transparency about individual data collection improves the efficacy of messages that are already individually focused - those with individual-good appeals - while the same transparency statement applied in a collectivist setting appears to conflict with people's sense of collective purpose. Relatedly, transparency regarding privacy and how individual data is protected in a collective setting may reduce concerns about personal privacy risk in a communal context, which prior work finds may be especially elevated, while the same transparency may be ineffective or even detrimental when placed in the context of individualistic privacy-benefit trade-offs [16].

Next, we consider demographic differences in responses to CovidDefense advertisements. Thus far it has been debated, on the basis of self-report evidence [17, 18, 19], whether men are less likely to adopt pro-social behaviors such as mask wearing. In this work we offer field evidence of a gender difference in receptiveness to COVID-19 pro-social messaging and behavior: men are significantly less likely to click on ads for CovidDefense (O.R. men $=0.794 ; p<0.001$; Appendix Table 6. We find that this effect varies in size based on the appeal shown in the ad. Both men and women consistently prefer collective-goods ads and men are less likely to click on both collective- and individual-goods ads. However, the gap in CTR between men and women is significantly larger for individual-goods ads: males click 23\% less often than females when shown individual-good ads compared to $10 \%$ less for collective-good ads (with $p<0.001$ for both; see Appendix Tables 7 and 8 .

Overall, we find that users between 34-64 are significantly less likely to click on the advertisements than those between 18 and 24 (O.R.s range from $0.874-0.951$ with $p<0.05$; see Figure 2c). On the other hand, those over 65 - who are also at high risk for developing and dying from COVID-19 [20]- are significantly more likely to click than those 18-24 (O.R. $=1.13 ; p<0.001)$. However, these effects are moderated both by the appeal of the ad shown and the gender of the ad viewer. Specifically, CTRs do not vary among different ages of women shown a collective-good ad (O.R.s range from 0.937-1.08 with $p>0.1$ ), likely because of the strength of this appeal for women, whom prior research shows are 


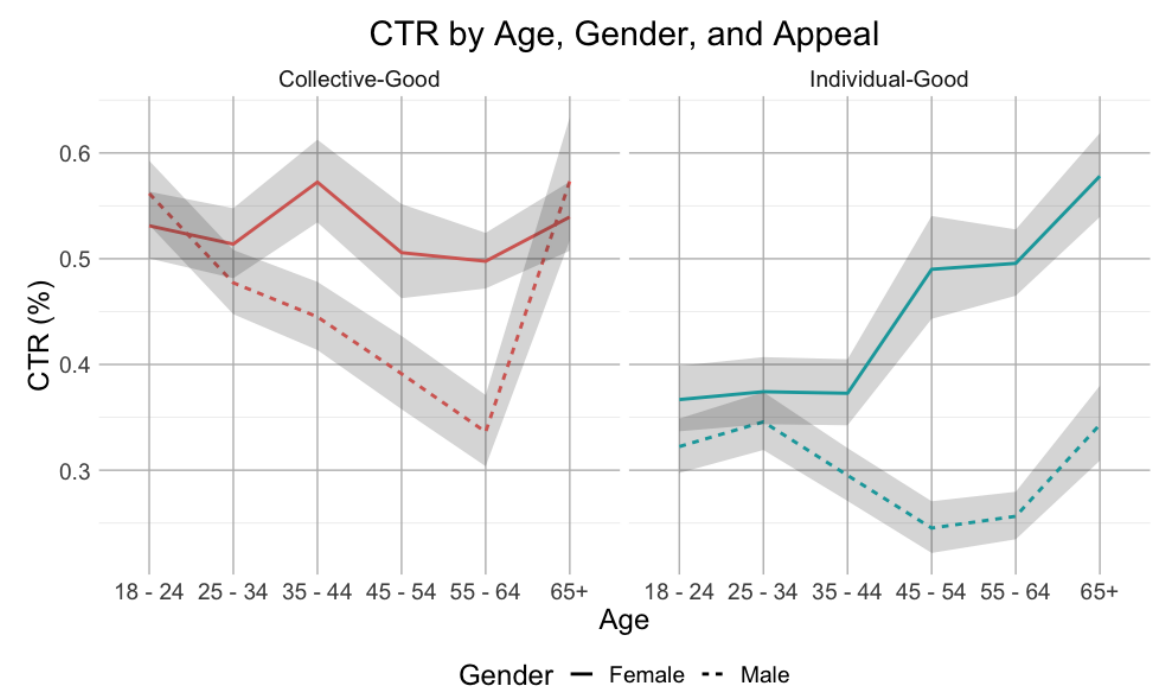

Figure 3: Click through rates (CTR) for each age group are reported with each curve representing an appeal and a gender. Collective-good appeals to females have no difference across the ages, whereas males see a significant drop in CTR in the middle ages. Individual-good appeals for females are non-decreasing across ages and generally flat for males. Regression tables are Appendix Table 7 and 8 . Error bars are 95\% confidence Clopper-Pearson intervals.

more relational - focused on the collective good - than men [21]. On the other hand, when shown an individual-good appeal, women 45 to $65+$ are significantly more likely to click than younger women (each age group above 45 sees an increase in CTR over the previous; O.R.s range from 1.34-1.58 with $p<0.001$ ). There is no difference between CTRs for ages 18-44 (O.R.s = 1.02 with $p>0.7$ ). We hypothesize that, aligned with [22], older women are cognizant of their higher COVID-19 risk and thus are willing to click even on an ad with an appeal that is less preferable and does not align with their broad tendency toward relationally-guided behavior.

Among men who were shown a collective-good appeal, young men (18-24 years old) and older men (65+ years old) are equally likely to click when shown a collective-good ad (O.R.=1.02 with $p=0.728)$, and are significantly more likely to click than middle aged men (O.R.s range from 0.597-0.849 with $p<0.001$ ). Considering individual-good ads, we observe a similar pattern, with no significant differences in the likelihood of clicking among men aged 18-44 and those over 65 (O.R.s range from 0.915-1.07 with $p>0.1$ ), while men aged 45-64 are significantly less likely to click on the same ads (O.R.s range from 0.760-0.785 with $p<0.001$ ). We find that men, especially those who are middle-aged and when presented with individual-good appeal, are far less likely than women to click to install CovidDefense. Prior literature finds that men have lower perceptions of their COVID-19 risk [22]. We hypothesize that the large gap in CTR between men and women, which is especially pronounced when presented with an individual-good appeal, is driven from the gender-based risk tolerance differences documented in the literature. When presented with an individual-good appeal that primes the viewer to especially focus on their own risk, the gender differences are even more pronounced.

Beyond the moderating effects of age and gender on the appeal used to advertise CovidDefense, we also find gender differences in the effect of our privacy and data transparency statements (see Table 1 ). While the effects shown in Figure $2 \mathrm{~b}$ are consistent across ages, we find that the overall effects of the privacy and data transparency statements are primarily driven by men's response to these statements. When facing collective-good appeal, men are more likely to click if presented with privacy controls of any sort, but are less likely to click if there is an explicit data transparency statement.

Women show different responses to the transparency statements. Specifically, when combined with a collective-good appeal, both privacy and data transparency statements have no impact on women's likelihood to click. Similar to the lack of age effect observed for women when presented with collective-good appeals, we hypothesize that there is such strong alignment between women's tendency toward relational choices and the collective-good appeal that other factors (e.g., age-specific risk perceptions, transparency statements) have no significant effect.

While both men and women are less likely to click on individual-good ads that include a technical privacy control, this reduced likelihood to click is larger among men (CI for O.R. for men: $(0.402,0.526)$; CI for O.R. for women: (0.738, 0.927)). Further, women are unaffected by the inclusion of a data-privacy transparency statement compared to men who are more likely to click ads with an individual-good appeal when a data transparency statement is included. Taken 
Table 1: Modeling the statement differences by Appeal and Gender

\begin{tabular}{|c|c|c|c|c|}
\hline & \multicolumn{4}{|c|}{ Dependent variable: } \\
\hline & \multicolumn{4}{|c|}{ Clicks } \\
\hline & \multicolumn{2}{|c|}{ Collective-Good } & \multicolumn{2}{|c|}{ Individual-Good } \\
\hline & $\begin{array}{l}\text { Male } \\
\text { (1) }\end{array}$ & $\begin{array}{c}\text { Female } \\
\text { (2) }\end{array}$ & $\begin{array}{l}\text { Male } \\
\text { (3) }\end{array}$ & $\begin{array}{c}\text { Female } \\
\text { (4) }\end{array}$ \\
\hline Privacy.Broad & $\begin{array}{c}\mathbf{1 . 1 6 0} \\
(1.049,1.283) \\
\mathrm{p}=0.004^{* *}\end{array}$ & $\begin{array}{c}0.993 \\
(0.906,1.087) \\
p=0.877\end{array}$ & $\begin{array}{c}\mathbf{0 . 7 9 6} \\
(0.707,0.896) \\
p=0.0002^{* *}\end{array}$ & $\begin{array}{c}\mathbf{1 . 1 9 8} \\
(1.076,1.335) \\
p=0.002^{* *}\end{array}$ \\
\hline NonTech.Control & $\begin{array}{c}\mathbf{1 . 1 1 0} \\
(1.009,1.222) \\
\mathrm{p}=0.032^{*}\end{array}$ & $\begin{array}{c}1.083 \\
(0.986,1.188) \\
\mathrm{p}=0.095\end{array}$ & $\begin{array}{c}1.018 \\
(0.899,1.152) \\
\mathrm{p}=0.784\end{array}$ & $\begin{array}{c}\mathbf{1 . 3 3 3} \\
(1.185,1.498) \\
\mathrm{p}=0.00001^{* *}\end{array}$ \\
\hline Technical.Control & $\begin{array}{c}\mathbf{1 . 2 3 6} \\
(1.118,1.366) \\
\mathrm{p}=0.00004^{* *}\end{array}$ & $\begin{array}{c}0.980 \\
(0.897,1.071) \\
\mathrm{p}=0.660\end{array}$ & $\begin{array}{c}\mathbf{0 . 4 6 0} \\
(0.402,0.526) \\
\mathrm{p}<0.001^{* *}\end{array}$ & $\begin{array}{c}\mathbf{0 . 8 2 7} \\
(0.738,0.927) \\
\mathrm{p}=0.002^{* *}\end{array}$ \\
\hline Data.Transparency & $\begin{array}{c}\mathbf{0 . 8 4 9} \\
(0.795,0.907) \\
p=0.00001^{* *}\end{array}$ & $\begin{array}{c}0.961 \\
(0.909,1.015) \\
\mathrm{p}=0.157\end{array}$ & $\begin{array}{c}\mathbf{1 . 2 7 5} \\
(1.178,1.379) \\
\mathrm{p}<0.001^{* *}\end{array}$ & $\begin{array}{c}1.058 \\
(0.987,1.134) \\
p=0.113\end{array}$ \\
\hline Constant & $\begin{array}{c}\mathbf{0 . 0 0 4} \\
(0.004,0.005) \\
\mathrm{p}<0.001^{* *}\end{array}$ & $\begin{array}{c}\mathbf{0 . 0 0 5} \\
(0.005,0.006) \\
\mathrm{p}<0.001^{* *}\end{array}$ & $\begin{array}{c}\mathbf{0 . 0 0 3} \\
(0.003,0.004) \\
\mathrm{p}<0.001^{* *}\end{array}$ & $\begin{array}{c}\mathbf{0 . 0 0 4} \\
(0.004,0.004) \\
p<0.001^{* *}\end{array}$ \\
\hline $\begin{array}{l}\text { Observations } \\
\text { Log Likelihood } \\
\text { Akaike Inf. Crit. }\end{array}$ & $\begin{array}{c}916,470 \\
-27,378.620 \\
54,767.230\end{array}$ & $\begin{array}{c}1,111,417 \\
-36,413.650 \\
72,837.310\end{array}$ & $\begin{array}{c}1,026,261 \\
-20,733.730 \\
41,477.460\end{array}$ & $\begin{array}{c}866,084 \\
-24,634.080 \\
49,278.150\end{array}$ \\
\hline
\end{tabular}

together, these findings - that women are affected positively by less technical statements of privacy while men are positively affected by technical and data related privacy statements - align with prior work finding that men and women focus on different privacy controls: men have been found to focus more on technical privacy controls, while women are more focused on privacy sentiment and non-technical controls [23, 24, 25, 26].

Finally, we examine the impact of geography on willingness to click on ads for CovidDefense. In contrast to the existing body of literature around urban-rural differences in COVID-19 behavior, which finds that rural residents are less concerned about COVID-19 and less likely to adopt pro-social COVID-19 behaviors [27, 28, 29, 30], we find that Louisiana residents in rural communities are significantly more likely to click on any of the proposed ads $(\mathrm{O} . \mathrm{R} .=1.15$; $p<0.001$; Appendix Table 6). This finding is robust across both appeals and all transparency statements (see Appendix Table 9).

\section{Conclusion}

This paper presents the findings from a randomized, large-scale field study, however, our work is not without limitations. Our results rely on a single study, from a single state (Louisiana) and with demographic data that rely on Google's ability to accurately classify gender, region and age. We encourage future studies to further investigate the impact of collective- vs. individual-goods appeals, as well as privacy and data transparency, on encouraging pro-social digital health behavior.

In sum, in a randomized field study, we find that residents of Louisiana are more likely to click on ads for exposure notification apps if the ad included a collective-good appeal. This effect was moderated (especially for men) by transparency regarding the individual data being collected and privacy protections offered for that data, likely due to the conflict between the sense of collective purpose and the associated cost for individual privacy. Moreover, we find gender 
and age differences in the likelihood to click the ads, fitting with past literature on gender differences and varying risks of COVID-19 across ages. These differences included lower probability to click on ads with technical privacy controls for women and higher likelihood for older people to click on ads due to the higher risk for COVID-19 associated with older people. We also find that the gender differences were made larger when the ads were individually-focused (with individual-good appeal), suggesting that the priming for individualism is enhancing gender differences. These findings may aid companies and policy makers when promoting digital tools to improve public health, especially those tools that have implications for privacy.

\section{Data Archival}

Data and analysis scripts for this experiment are available in an anonymized form at https://doi.org/10.7910/ DVN/MLUR6D

\section{Contributions}

E.M.R. and D.T. designed the study. E.M.R. recruited and coordinated with the Louisiana Department of Health. S.D., J.D., and E.M.R. ran the field experiment on Google Ads. S.D. and E.M.R. analyzed the data. S.D., J.D., E.M.R., and D.T. wrote the paper.

\section{Acknowledgements}

The authors thank the Louisiana Department of Health and especially: Jay Besse, DeAnn Gruber, Shelly Gulati, Rachael Kramas, Lee Mendoza, Scott Teesdale. We additionally thank ThreeSixtyEight, especially Jeremy Beyt and Gus Murillo, and the PathCheck Foundation, especially Mary-Katherine McCarey and Ramesh Raskar. The Max Planck Institute for Software Systems funded the advertisements used in this study. Dickerson and Dooley were supported in part by NSF CAREER Award IIS-1846237, NIST MSE Award \#20126334, DARPA GARD \#HR00112020007, DARPA SI3-CMD \#S4761, and DoD WHS Award \#HQ003420F0035.

\section{References}

[1] National Academies of Sciences, Engineering, and Medicine and others. Encouraging adoption of protective behaviors to mitigate the spread of covid-19: Strategies for behavior change (2020).

[2] Rabb, N., Glick, D., Houston, A., Bowers, J. \& Yokum, D. No evidence that collective-good appeals best promote covid-related health behaviors. Proceedings of the National Academy of Sciences 118 (2021).

[3] Korn, L., Böhm, R., Meier, N. W. \& Betsch, C. Vaccination as a social contract. Proceedings of the National Academy of Sciences 117, 14890-14899 (2020).

[4] Munzert, S., Selb, P., Gohdes, A., Stoetzer, L. F. \& Lowe, W. Tracking and promoting the usage of a covid-19 contact tracing app. Nature Human Behaviour 5, 247-255 (2021).

[5] Toussaert, S. Upping uptake of covid contact tracing apps. Nature Human Behaviour 5, 183-184 (2021).

[6] Banker, S. \& Park, J. Evaluating prosocial covid-19 messaging frames: Evidence from a field study on facebook. Judgment and Decision Making 15, 1037-1043 (2020).

[7] Zhang, B., Kreps, S., McMurry, N. \& McCain, R. M. Americans' perceptions of privacy and surveillance in the covid-19 pandemic. Plos one 15, e0242652 (2020).

[8] Gefen, G., Ben-Porat, O., Tennenholtz, M. \& Yom-Tov, E. Privacy, altruism, and experience: Estimating the perceived value of internet data for medical uses. In Companion Proceedings of the Web Conference 2020, 552-556 (2020).

[9] Tucker, C. E. Social networks, personalized advertising, and privacy controls. Journal of marketing research 51, 546-562 (2014).

[10] Schaub, F., Balebako, R. \& Cranor, L. F. Designing effective privacy notices and controls. IEEE Internet Computing (2017).

[11] Bandy, J. \& Hecht, B. Errors in geotargeted display advertising: Good news for local journalism? Proceedings of the ACM on Human-Computer Interaction 5 (2021). 
[12] McDonald, P., Mohebbi, M. \& Slatkin, B. Comparing google consumer surveys to existing probability and non-probability based internet surveys. Google White Paper (2012).

[13] Allik, J. \& Realo, A. Individualism-collectivism and social capital. Journal of cross-cultural psychology 35, 29-49 (2004).

[14] Baldassarri, D. \& Abascal, M. Diversity and prosocial behavior. Science 369, 1183-1187 (2020).

[15] Kemmelmeier, M., Jambor, E. E. \& Letner, J. Individualism and good works: Cultural variation in giving and volunteering across the united states. Journal of Cross-Cultural Psychology 37, 327-344 (2006).

[16] Trepte, S. et al. A cross-cultural perspective on the privacy calculus. Social Media+ Society 3, 2056305116688035 (2017).

[17] White, A. Men and covid-19: the aftermath. Postgraduate Medicine 132, 18-27 (2020).

[18] Barber, S. J. \& Kim, H. Covid-19 worries and behavior changes in older and younger men and women. The Journals of Gerontology: Series B 76, e17-e23 (2021).

[19] Cassino, D. \& Besen-Cassino, Y. Of masks and men? gender, sex, and protective measures during covid-19. Politics \& Gender 16, 1052-1062 (2020).

[20] for Disease Control, C., Prevention et al. Older adult at greater risk of requiring hospitalization or dying if diagnosed with covid-19 (2020).

[21] Kashima, Y. et al. Culture, gender, and self: a perspective from individualism-collectivism research. Journal of personality and social psychology 69, 925 (1995).

[22] Fan, Y., Orhun, A. Y. \& Turjeman, D. Heterogeneous actions, beliefs, constraints and risk tolerance during the covid-19 pandemic. Tech. Rep., National Bureau of Economic Research (2020).

[23] Habib, H. et al. User behaviors and attitudes under password expiration policies. In Fourteenth Symposium on Usable Privacy and Security ( $\{$ SOUPS $\}$ 2018), 13-30 (2018).

[24] Kuo, F.-Y., Lin, C. S. \& Hsu, M.-H. Assessing gender differences in computer professionals' self-regulatory efficacy concerning information privacy practices. Journal of business ethics 73, 145-160 (2007).

[25] Mathur, A., Vitak, J., Narayanan, A. \& Chetty, M. Characterizing the use of browser-based blocking extensions to prevent online tracking. In Fourteenth Symposium on Usable Privacy and Security ( $\{$ SOUPS $\} 2018), 103-116$ (2018).

[26] Redmiles, E. Net benefits: Digital inequities in social capital, privacy preservation, and digital parenting practices of us social media users. In Proceedings of the International AAAI Conference on Web and Social Media, vol. 12 (2018).

[27] Callaghan, T., Lueck, J. A., Trujillo, K. L. \& Ferdinand, A. O. Rural and urban differences in covid-19 prevention behaviors. The Journal of Rural Health (2021).

[28] Chen, X. \& Chen, H. Differences in preventive behaviors of covid-19 between urban and rural residents: lessons learned from a cross-sectional study in china. International journal of environmental research and public health 17, 4437 (2020).

[29] Huang, Q. et al. Urban-rural differences in covid-19 exposures and outcomes in the south: A preliminary analysis of south carolina. PloS one 16, e0246548 (2021).

[30] Haischer, M. H. et al. Who is wearing a mask? gender-, age-, and location-related differences during the covid-19 pandemic. PloS one 15, e0240785 (2020). 


\section{Appendix}

\section{A Advertisements}

The full text of the 14 ad campaigns are included here for clarity:

1. Get notified of COVID exposure.

2. Get notified of COVID exposure, without harming your privacy.

3. Get notified of COVID exposure. App data stays on your device.

4. Get notified of COVID exposure. You control the data you share.

5. Get notified of COVID exposure. CovidDefense uses information about who you have been near, without harming your privacy.

6. Get notified of COVID exposure. CovidDefense uses information about who you have been near. App data stays on your device.

7. Get notified of COVID exposure. CovidDefense uses information about who you have been near. You control the data you share.

8. Reduce COVID infections.

9. Reduce COVID infections, without harming your privacy.

10. Reduce COVID infections. App data stays on your device.

11. Reduce COVID infections. You control the data you share.

12. Reduce COVID infections. The app uses information about who you have been near, without harming your privacy.

13. Reduce COVID infections. The app uses information about who you have been near. App data stays on your device.

14. Reduce COVID infections. The app uses information about who you have been near. You control the data you share.

An example advertisement (for Ad \#1) can be found in Figure 4

\section{B Datasets}

The entire dataset and model regressions can be found here: https://doi.org/10.7910/DVN/MLUR6D

There are two primary datasets: one which has all 7,010,271 impressions and demographic data, and another with just the impressions that have associated geographic information. The former includes columns for Google-estimated demographics like Age and Gender, with many impressions having values of "Unknown".

These two data tables for demographic and geographic impressions were represented by a row for each impression with columns for whether that impression resulted in a click; the age and gender or geography of the impression; as well as indicator variables for the presence or absence of ad information (appeals, privacy transparency - broad privacy reassurance, non-technical control, and technical control - and data transparency).

An associated R file is included which includes functions to reproduce each model and associated statistics.

\section{Regressions}

We report the full regression tables for all claims made in the paper.

Table 2 includes overall effects for each experimental variable. These regressions are performed with all the data, just demographic data, and just geographic data.

Table 3 reports the interaction effect between each privacy and transparency statement with the two appeals. These regressions are performed with all the data, just demographic data, and just geographic data.

Table 4 reports the regression for each statement for all collective-good ads. These regressions are performed with all the data, just demographic data, and just geographic data. 
Example of your image ad at $160 \times 600$

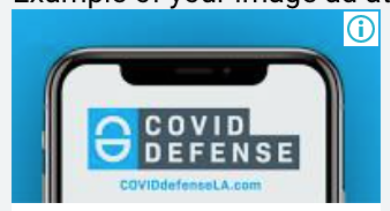

Get

notified of

covid

exposure

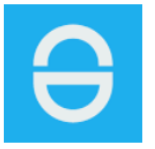

Install

CovidDefense

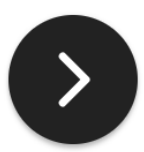

Example of your image ad at $300 \times 250$

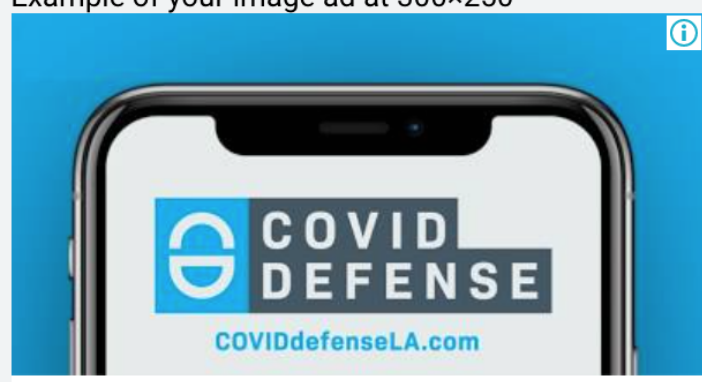

Get notified of covid exposure

Install CovidDefense

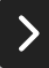

Example of your text ad at $300 \times 250$

(i)

\section{Get notified of covid exposure}

CovidDefense

Install CovidDefense

\section{OPEN}

Example of your native ad at $480 \times 120$

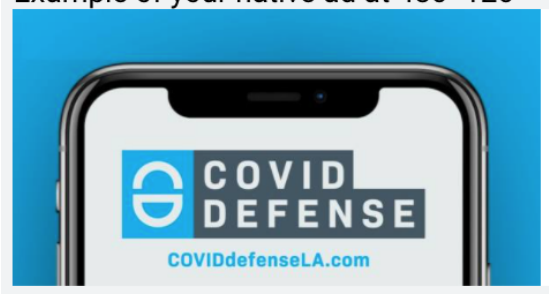

Get notified of covid

(i) exposure

Ad Install CovidDefense

CovidDefense

Figure 4: Four examples of how Ad \#1 is displayed on a computer. 
Table 5 reports the regression for each statement for all individual-good ads. These regressions are performed with all the data, just demographic data, and just geographic data.

Table 6 reports the regression for each experimental, demographic, and geographic variable.

Table 7 reports the regressions for age and gender differences for collective-good ads.

Table 8 reports the regressions for age and gender differences for individual-good ads.

Table 9 reports the regressions for Urban/Rural interactions with the experimental variables.

Table 2: Modeling the five independent variables

\begin{tabular}{|c|c|c|c|}
\hline & \multicolumn{3}{|c|}{ Dependent variable: } \\
\hline & $\begin{array}{l}\text { All data } \\
\text { (1) }\end{array}$ & $\begin{array}{c}\text { Clicks } \\
\text { Just Demographic } \\
(2) \\
\end{array}$ & $\begin{array}{c}\text { Just Geographic } \\
\text { (3) }\end{array}$ \\
\hline Individual.Good & $\begin{array}{c}0.745 \\
(0.727,0.763) \\
p<0.001^{* *}\end{array}$ & $\begin{array}{c}0.730 \\
(0.707,0.752) \\
p<0.001^{* *}\end{array}$ & $\begin{array}{c}0.744 \\
(0.726,0.762) \\
p<0.001^{* *}\end{array}$ \\
\hline Privacy.Broad & $\begin{array}{c}1.032 \\
(0.992,1.074) \\
p=0.121\end{array}$ & $\begin{array}{c}1.031 \\
(0.980,1.085) \\
p=0.243\end{array}$ & $\begin{array}{c}1.034 \\
(0.993,1.076) \\
p=0.106\end{array}$ \\
\hline NonTech.Control & $\begin{array}{c}1.084 \\
(1.042,1.128) \\
p=0.0001^{* *}\end{array}$ & $\begin{array}{c}1.112 \\
(1.056,1.172) \\
p=0.0001^{* *}\end{array}$ & $\begin{array}{c}1.092 \\
(1.049,1.137) \\
\mathrm{p}=0.00002^{* *}\end{array}$ \\
\hline Technical.Control & $\begin{array}{c}0.920 \\
(0.883,0.958) \\
p=0.0001^{* *}\end{array}$ & $\begin{array}{c}0.890 \\
(0.844,0.938) \\
\mathrm{p}=0.00002^{* *}\end{array}$ & $\begin{array}{c}0.924 \\
(0.886,0.963) \\
p=0.0002^{* *}\end{array}$ \\
\hline Data.Transparency & $\begin{array}{c}0.981 \\
(0.957,1.007) \\
p=0.147\end{array}$ & $\begin{array}{c}1.028 \\
(0.995,1.062) \\
p=0.100\end{array}$ & $\begin{array}{c}0.977 \\
(0.953,1.003) \\
p=0.081\end{array}$ \\
\hline Constant & $\begin{array}{c}0.005 \\
(0.004,0.005) \\
\mathrm{p}<0.001^{* *}\end{array}$ & $\begin{array}{c}0.005 \\
(0.005,0.005) \\
p<0.001^{* *}\end{array}$ & $\begin{array}{c}0.005 \\
(0.004,0.005) \\
\mathrm{p}<0.001^{* *}\end{array}$ \\
\hline $\begin{array}{l}\text { Observations } \\
\text { Log Likelihood } \\
\text { Akaike Inf. Crit. }\end{array}$ & $\begin{array}{c}7,010,271 \\
-182,372.500 \\
364,756.900 \\
\end{array}$ & $\begin{array}{c}3,920,232 \\
-109,490.000 \\
218,992.000\end{array}$ & $\begin{array}{c}6,858,820 \\
-178,407.300 \\
356,826.500 \\
\end{array}$ \\
\hline
\end{tabular}


Table 3: Modeling the interaction of the appeal with the privacy and transparency statements

\begin{tabular}{|c|c|c|c|}
\hline & \multicolumn{3}{|c|}{ Dependent variable: } \\
\hline & $\begin{array}{l}\text { All data } \\
\text { (1) }\end{array}$ & $\begin{array}{c}\text { Clicks } \\
\text { Just Demographic } \\
(2)\end{array}$ & $\begin{array}{c}\text { Just Geographic } \\
\text { (3) }\end{array}$ \\
\hline Individual.Good & $\begin{array}{c}0.880 \\
(0.827,0.937) \\
p=0.0001^{* *}\end{array}$ & $\begin{array}{c}0.763 \\
(0.703,0.829) \\
p<0.001^{* *}\end{array}$ & $\begin{array}{c}0.878 \\
(0.824,0.935) \\
p=0.0001^{* *}\end{array}$ \\
\hline Privacy.Broad & $\begin{array}{c}1.106 \\
(1.048,1.167) \\
p=0.0003^{* *}\end{array}$ & $\begin{array}{c}1.073 \\
(1.003,1.147) \\
p=0.041^{*}\end{array}$ & $\begin{array}{c}1.110 \\
(1.052,1.172) \\
\mathrm{p}=0.0002^{* *}\end{array}$ \\
\hline NonTech.Control & $\begin{array}{c}1.123 \\
(1.064,1.185) \\
p=0.00003^{* *}\end{array}$ & $\begin{array}{c}1.096 \\
(1.026,1.172) \\
p=0.007^{* *}\end{array}$ & $\begin{array}{c}1.131 \\
(1.071,1.195) \\
\mathrm{p}=0.00001^{* *}\end{array}$ \\
\hline Technical.Control & $\begin{array}{c}1.203 \\
(1.140,1.269) \\
p<0.001^{* *}\end{array}$ & $\begin{array}{c}1.095 \\
(1.025,1.169) \\
p=0.008^{* *}\end{array}$ & $\begin{array}{c}1.209 \\
(1.146,1.277) \\
p<0.001^{* *}\end{array}$ \\
\hline Data.Transparency & $\begin{array}{c}0.911 \\
(0.881,0.942) \\
\mathrm{p}<0.00001^{* *}\end{array}$ & $\begin{array}{c}0.913 \\
(0.875,0.952) \\
p=0.00003^{* *}\end{array}$ & $\begin{array}{c}0.904 \\
(0.875,0.935) \\
p<0.001^{* *}\end{array}$ \\
\hline Individual.Good:Data.Transparency & $\begin{array}{c}1.185 \\
(1.126,1.248) \\
p<0.001^{* *}\end{array}$ & $\begin{array}{c}1.280 \\
(1.197,1.369) \\
p<0.001^{* *}\end{array}$ & $\begin{array}{c}1.194 \\
(1.134,1.258) \\
p<0.001^{* *}\end{array}$ \\
\hline Individual.Good:Privacy.Broad & $\begin{array}{c}0.855 \\
(0.789,0.927) \\
p=0.0002^{* *}\end{array}$ & $\begin{array}{c}0.910 \\
(0.820,1.009) \\
p=0.075\end{array}$ & $\begin{array}{c}0.851 \\
(0.785,0.923) \\
\mathrm{p}=0.0002^{* *}\end{array}$ \\
\hline Individual.Good:NonTech.Control & $\begin{array}{c}0.914 \\
(0.843,0.990) \\
p=0.028^{*}\end{array}$ & $\begin{array}{c}1.041 \\
(0.934,1.160) \\
p=0.469\end{array}$ & $\begin{array}{c}0.914 \\
(0.843,0.991) \\
p=0.030^{*}\end{array}$ \\
\hline Individual.Good:Technical.Control & $\begin{array}{c}0.515 \\
(0.473,0.559) \\
\mathrm{p}<0.001^{* *}\end{array}$ & $\begin{array}{c}0.581 \\
(0.521,0.648) \\
\mathrm{p}<0.001^{* *}\end{array}$ & $\begin{array}{c}0.514 \\
(0.472,0.559) \\
p<0.001^{* *}\end{array}$ \\
\hline Constant & $\begin{array}{c}0.004 \\
(0.004,0.004) \\
p<0.001^{* *}\end{array}$ & $\begin{array}{c}0.005 \\
(0.005,0.005) \\
p<0.001^{* *}\end{array}$ & $\begin{array}{c}0.004 \\
(0.004,0.004) \\
p<0.001^{* *}\end{array}$ \\
\hline $\begin{array}{l}\text { Observations } \\
\text { Log Likelihood } \\
\text { Akaike Inf. Crit. }\end{array}$ & $\begin{array}{c}7,010,271 \\
-182,159.100 \\
364,338.100 \\
\end{array}$ & $\begin{array}{c}3,920,232 \\
-109,357.700 \\
218,735.300 \\
\end{array}$ & $\begin{array}{c}6,858,820 \\
-178,196.900 \\
356,413.700 \\
\end{array}$ \\
\hline
\end{tabular}


Table 4: Modeling the privacy and transparency statements for Collective-Good ads

\begin{tabular}{lccc}
\hline \hline & \multicolumn{3}{c}{ Dependent variable: } \\
\cline { 2 - 4 } & All data & Clicks & \\
& $(1)$ & $(2)$ & $(3)$ \\
\hline Privacy.Broad & 1.106 & 1.073 & 1.110 \\
& $(1.048,1.167)$ & $(1.003,1.147)$ & $(1.052,1.172)$ \\
& $\mathrm{p}=0.0003^{* *}$ & $\mathrm{p}=0.041^{*}$ & $\mathrm{p}=0.0002^{* *}$ \\
NonTech.Control & 1.123 & 1.096 & 1.131 \\
& $(1.064,1.185)$ & $(1.026,1.172)$ & $(1.071,1.195)$ \\
& $\mathrm{p}=0.00003^{* *}$ & $\mathrm{p}=0.007^{* *}$ & $\mathrm{p}=0.00001^{* *}$ \\
Technical.Control & 1.203 & 1.095 & 1.209 \\
& $(1.140,1.269)$ & $(1.025,1.169)$ & $(1.146,1.277)$ \\
& $\mathrm{p}<0.001^{* *}$ & $\mathrm{p}=0.008^{* *}$ & $\mathrm{p}<0.001^{* *}$ \\
Data.Transparency & 0.911 & 0.913 & 0.904 \\
& $(0.881,0.942)$ & $(0.875,0.952)$ & $(0.875,0.935)$ \\
& $\mathrm{p}<0.00001^{* *}$ & $\mathrm{p}=0.00003^{* *}$ & $\mathrm{p}<0.001^{* *}$ \\
Constant & & & \\
& 0.004 & 0.005 & 0.004 \\
& $(0.004,0.004)$ & $(0.005,0.005)$ & $(0.004,0.004)$ \\
\hline Observations & $\mathrm{p}<0.001^{* *}$ & $\mathrm{p}<0.001^{* *}$ & $\mathrm{p}<0.001^{* *}$ \\
Log Likelihood & & & \\
Akaike Inf. Crit. & $205,523,339$ & $2,027,887$ & $3,446,697$ \\
\hline Note: & 205.9000 & $-63,818.110$ & $-100,767.200$ \\
& & $127,646.200$ & $201,544.400$ \\
\hline
\end{tabular}


Table 5: Modeling the privacy and transparency statements for Individual-Good ads

\begin{tabular}{lccc}
\hline \hline & \multicolumn{3}{c}{ Dependent variable: } \\
\cline { 2 - 4 } & All data & Clicks & \\
& $(1)$ & $(2)$ & $(3)$ \\
\hline Privacy.Broad & 0.946 & 0.976 & 0.945 \\
& $(0.891,1.004)$ & $(1.003,1.147)$ & $(0.890,1.004)$ \\
& $\mathrm{p}=0.070$ & $\mathrm{p}=0.547$ & $\mathrm{p}=0.068$ \\
NonTech.Control & 1.026 & 1.141 & 1.034 \\
& $(0.967,1.089)$ & $(1.026,1.172)$ & $(0.974,1.098)$ \\
& $\mathrm{p}=0.396$ & $\mathrm{p}=0.003^{* *}$ & $\mathrm{p}=0.274$ \\
Technical.Control & 0.619 & 0.636 & 0.621 \\
& $(0.581,0.660)$ & $(1.025,1.169)$ & $(0.582,0.663)$ \\
& $\mathrm{p}<0.001^{* *}$ & $\mathrm{p}<0.001^{* *}$ & $\mathrm{p}<0.001^{* *}$ \\
Data.Transparency & 1.080 & 1.169 & 1.080 \\
& $(1.038,1.123)$ & $(0.875,0.952)$ & $(1.038,1.124)$ \\
& $\mathrm{p}=0.0002^{* *}$ & $\mathrm{p}<0.001^{* *}$ & $\mathrm{p}=0.0002^{* *}$ \\
Constant & 0.004 & 0.004 & 0.004 \\
& $(0.004,0.004)$ & $(0.005,0.005)$ & $(0.004,0.004)$ \\
& $\mathrm{p}<0.001^{* *}$ & $\mathrm{p}<0.001^{* *}$ & $\mathrm{p}<0.001^{* *}$ \\
\hline Observations & $3,486,932$ & $1,892,345$ & $3,412,123$ \\
Log Likelihood & $-79,213.640$ & $-45,539.550$ & $-77,429.660$ \\
Akaike Inf. Crit. & $158,437.300$ & $91,089.100$ & $154,869.300$ \\
\hline \hline Note: & & & ${ }^{*} \mathrm{p}<0.05 ;{ }^{* *} \mathrm{p}<0.01$ \\
& & &
\end{tabular}


Table 6: Modeling demographics and geographics

\begin{tabular}{|c|c|c|}
\hline & \multicolumn{2}{|c|}{ Dependent variable: } \\
\hline & \multicolumn{2}{|c|}{ Clicks } \\
\hline & $\begin{array}{c}\text { Just Demographic } \\
\text { (1) }\end{array}$ & $\begin{array}{c}\text { Just Geographic } \\
\text { (2) }\end{array}$ \\
\hline Age25 - 34 & $\begin{array}{c}0.951 \\
(0.906,0.998) \\
\mathrm{p}=0.041^{*}\end{array}$ & \\
\hline Age $35-44$ & $\begin{array}{c}0.932 \\
(0.886,0.980) \\
p=0.006^{* *}\end{array}$ & \\
\hline Age45 - 54 & $\begin{array}{c}0.874 \\
(0.825,0.925) \\
p=0.00001^{* *}\end{array}$ & \\
\hline Age55 - 64 & $\begin{array}{c}0.909 \\
(0.866,0.954) \\
\mathrm{p}=0.0001^{* *}\end{array}$ & \\
\hline Age65+ & $\begin{array}{c}1.134 \\
(1.077,1.194) \\
\mathrm{p}=0.00001^{* *}\end{array}$ & \\
\hline GenderMale & $\begin{array}{c}0.794 \\
(0.769,0.819) \\
p<0.001^{* *}\end{array}$ & \\
\hline DensityRural & & $\begin{array}{c}1.146 \\
(1.104,1.189) \\
p<0.001^{* *}\end{array}$ \\
\hline Individual.Good & $\begin{array}{c}0.747 \\
(0.724,0.770) \\
p<0.001^{* *}\end{array}$ & $\begin{array}{c}0.744 \\
(0.726,0.762) \\
\mathrm{p}<0.001^{* *}\end{array}$ \\
\hline Privacy.Broad & $\begin{array}{c}1.014 \\
(0.964,1.068) \\
p=0.584\end{array}$ & $\begin{array}{c}1.033 \\
(0.993,1.076) \\
p=0.110\end{array}$ \\
\hline NonTech.Control & $\begin{array}{c}1.115 \\
(1.058,1.175) \\
p=0.00005^{* *}\end{array}$ & $\begin{array}{c}1.091 \\
(1.048,1.136) \\
p=0.00003^{* *}\end{array}$ \\
\hline Technical.Control & $\begin{array}{c}0.857 \\
(0.813,0.903) \\
\mathrm{p}<0.001^{* *}\end{array}$ & $\begin{array}{c}0.923 \\
(0.886,0.962) \\
\mathrm{p}=0.0002^{* *}\end{array}$ \\
\hline Data.Transparency & $\begin{array}{c}1.034 \\
(1.001,1.068) \\
\mathrm{p}=0.047^{*}\end{array}$ & $\begin{array}{c}0.978 \\
(0.953,1.003) \\
p=0.084\end{array}$ \\
\hline Constant & $\begin{array}{c}0.006 \\
(0.005,0.006) \\
p<0.001^{* *}\end{array}$ & $\begin{array}{c}0.005 \\
(0.004,0.005) \\
p<0.001^{* *}\end{array}$ \\
\hline $\begin{array}{l}\text { Observations } \\
\text { Log Likelihood } \\
\text { Akaike Inf. Crit. }\end{array}$ & $\begin{array}{c}3,920,232 \\
-109,314.800 \\
218,653.500\end{array}$ & $\begin{array}{c}6,858,820 \\
-178,382.400 \\
356,778.700\end{array}$ \\
\hline
\end{tabular}


Table 7: Modeling the Age and Gender differences for Collective-Good ads

\begin{tabular}{|c|c|c|c|}
\hline & \multicolumn{3}{|c|}{ Dependent variable: } \\
\hline & $\begin{array}{c}\text { Collective-Good } \\
\text { (1) }\end{array}$ & $\begin{array}{c}\text { Clicks } \\
\text { Collective-Good Female } \\
(2)\end{array}$ & $\begin{array}{c}\text { Collective-Good Male } \\
(3)\end{array}$ \\
\hline Age25-34 & $\begin{array}{c}0.902 \\
(0.849,0.958) \\
\mathrm{p}=0.001^{* *}\end{array}$ & $\begin{array}{c}0.967 \\
(0.886,1.055) \\
p=0.455\end{array}$ & $\begin{array}{c}0.849 \\
(0.781,0.923) \\
p=0.0002^{* *}\end{array}$ \\
\hline Age35 - 44 & $\begin{array}{c}0.921 \\
(0.864,0.981) \\
\mathrm{p}=0.012^{*}\end{array}$ & $\begin{array}{c}1.078 \\
(0.985,1.180) \\
p=0.103\end{array}$ & $\begin{array}{c}0.791 \\
(0.723,0.866) \\
p<0.00001^{* *}\end{array}$ \\
\hline Age45 - 54 & $\begin{array}{c}0.808 \\
(0.751,0.870) \\
\mathrm{p}<0.00001^{* *}\end{array}$ & $\begin{array}{c}0.952 \\
(0.856,1.058) \\
p=0.360\end{array}$ & $\begin{array}{c}0.695 \\
(0.627,0.771) \\
\mathrm{p}<0.001^{* *}\end{array}$ \\
\hline Age55 - 64 & $\begin{array}{c}0.798 \\
(0.750,0.850) \\
\mathrm{p}<0.001^{* *}\end{array}$ & $\begin{array}{c}0.937 \\
(0.865,1.014) \\
p=0.106\end{array}$ & $\begin{array}{c}0.597 \\
(0.533,0.669) \\
\mathrm{p}<0.001^{* *}\end{array}$ \\
\hline Age65+ & $\begin{array}{c}0.969 \\
(0.906,1.035) \\
\mathrm{p}=0.344\end{array}$ & $\begin{array}{c}1.016 \\
(0.933,1.105) \\
p=0.718\end{array}$ & $\begin{array}{c}1.021 \\
(0.911,1.144) \\
p=0.728\end{array}$ \\
\hline GenderMale & $\begin{array}{c}0.887 \\
(0.851,0.924) \\
\mathrm{p}<0.001^{* *}\end{array}$ & & \\
\hline Constant & $\begin{array}{c}0.006 \\
(0.006,0.006) \\
\mathrm{p}<0.001^{* *}\end{array}$ & $\begin{array}{c}0.005 \\
(0.005,0.006) \\
\mathrm{p}<0.001^{* *}\end{array}$ & $\begin{array}{c}0.006 \\
(0.005,0.006) \\
\mathrm{p}<0.001^{* *}\end{array}$ \\
\hline $\begin{array}{l}\text { Observations } \\
\text { Log Likelihood } \\
\text { Akaike Inf. Crit. }\end{array}$ & $\begin{array}{c}2,027,887 \\
-63,777.320 \\
127,568.600 \\
\end{array}$ & $\begin{array}{c}1,111,417 \\
-36,412.990 \\
72,837.980 \\
\end{array}$ & $\begin{array}{c}916,470 \\
-27,332.210 \\
54,676.420\end{array}$ \\
\hline
\end{tabular}


Table 8: Modeling the Age and Gender differences for Individual-Good ads

\begin{tabular}{|c|c|c|c|}
\hline & \multicolumn{3}{|c|}{ Dependent variable: } \\
\hline & $\begin{array}{c}\text { Individual-Good } \\
\text { (1) }\end{array}$ & $\begin{array}{c}\text { Clicks } \\
\text { Individual-Good Female } \\
(2)\end{array}$ & $\begin{array}{c}\text { Individual-Good Male } \\
\text { (3) }\end{array}$ \\
\hline Age25-34 & $\begin{array}{c}1.046 \\
(0.964,1.134) \\
p=0.280\end{array}$ & $\begin{array}{c}1.020 \\
(0.906,1.149) \\
p=0.740\end{array}$ & $\begin{array}{c}1.073 \\
(0.959,1.199) \\
p=0.218\end{array}$ \\
\hline Age $35-44$ & $\begin{array}{c}0.962 \\
(0.886,1.045) \\
\mathrm{p}=0.357\end{array}$ & $\begin{array}{c}1.016 \\
(0.903,1.144) \\
p=0.788\end{array}$ & $\begin{array}{c}0.915 \\
(0.816,1.027) \\
\mathrm{p}=0.133\end{array}$ \\
\hline Age45 - 54 & $\begin{array}{c}0.997 \\
(0.911,1.091) \\
\mathrm{p}=0.947\end{array}$ & $\begin{array}{c}1.337 \\
(1.175,1.522) \\
p=0.00002^{* *}\end{array}$ & $\begin{array}{c}0.760 \\
(0.670,0.863) \\
p=0.00003^{* *}\end{array}$ \\
\hline Age55 - 64 & $\begin{array}{c}1.074 \\
(0.995,1.160) \\
\mathrm{p}=0.068\end{array}$ & $\begin{array}{c}1.353 \\
(1.218,1.502) \\
p<0.00001^{* *}\end{array}$ & $\begin{array}{c}0.795 \\
(0.707,0.894) \\
p=0.0002^{* *}\end{array}$ \\
\hline Age65+ & $\begin{array}{c}1.331 \\
(1.227,1.443) \\
\mathrm{p}<0.001^{* *}\end{array}$ & $\begin{array}{c}1.580 \\
(1.418,1.760) \\
\mathrm{p}<0.001^{* *}\end{array}$ & $\begin{array}{c}1.065 \\
(0.936,1.211) \\
p=0.342\end{array}$ \\
\hline GenderMale & $\begin{array}{c}0.685 \\
(0.653,0.719) \\
\mathrm{p}<0.001^{* *}\end{array}$ & & \\
\hline Constant & $\begin{array}{c}0.004 \\
(0.004,0.004) \\
\mathrm{p}<0.001^{* *}\end{array}$ & $\begin{array}{c}0.004 \\
(0.003,0.004) \\
\mathrm{p}<0.001^{* *}\end{array}$ & $\begin{array}{c}0.003 \\
(0.003,0.004) \\
\mathrm{p}<0.001^{* *}\end{array}$ \\
\hline $\begin{array}{l}\text { Observations } \\
\text { Log Likelihood } \\
\text { Akaike Inf. Crit. }\end{array}$ & $\begin{array}{c}1,892,345 \\
-45,551.090 \\
91,116.180\end{array}$ & $\begin{array}{c}866,084 \\
-24,641.040 \\
49,294.090\end{array}$ & $\begin{array}{c}1,026,261 \\
-20,858.890 \\
41,729.780\end{array}$ \\
\hline
\end{tabular}


Table 9: Modeling the statement differences with an interaction for Density

\begin{tabular}{|c|c|c|c|c|c|}
\hline & \multicolumn{5}{|c|}{ Dependent variable: } \\
\hline & $\begin{array}{l}\text { Appeal } \\
\text { (1) }\end{array}$ & $\begin{array}{l}\text { Privacy: Broad } \\
\text { (2) }\end{array}$ & $\begin{array}{c}\text { Clicks } \\
\text { Non-Technical Control } \\
\text { (3) }\end{array}$ & $\begin{array}{c}\text { Technical Control } \\
\text { (4) }\end{array}$ & $\begin{array}{c}\text { Data Transparency } \\
\text { (5) }\end{array}$ \\
\hline DensityRural & $\begin{array}{c}1.114 \\
(1.061,1.170) \\
\mathrm{p}=0.00002^{* *}\end{array}$ & $\begin{array}{c}1.132 \\
(1.082,1.183) \\
\mathrm{p}<0.00001^{* *}\end{array}$ & $\begin{array}{c}1.164 \\
(1.113,1.217) \\
\mathrm{p}<0.0001^{* *}\end{array}$ & $\begin{array}{c}1.151 \\
(1.103,1.202) \\
\mathrm{p}<0.0001^{* *}\end{array}$ & $\begin{array}{c}1.158 \\
(1.103,1.216) \\
\mathrm{p}<0.0001^{* *}\end{array}$ \\
\hline Individual.Good & $\begin{array}{c}0.738 \\
(0.719,0.757) \\
\mathrm{p}<0.0001^{* *}\end{array}$ & & & & \\
\hline DensityRural:Individual.Good & $\begin{array}{c}1.067 \\
(0.990,1.150) \\
\mathrm{p}=0.090\end{array}$ & & & & \\
\hline Privacy.Broad & & $\begin{array}{c}1.025 \\
(0.997,1.054) \\
p=0.076\end{array}$ & & & \\
\hline DensityRural:Privacy.Broad & & $\begin{array}{c}1.055 \\
(0.973,1.143) \\
p=0.197\end{array}$ & & & \\
\hline NonTech.Control & & & $\begin{array}{c}1.110 \\
(1.080,1.141) \\
\mathrm{p}<0.0001^{* *}\end{array}$ & & \\
\hline DensityRural:NonTech.Control & & & $\begin{array}{c}0.959 \\
(0.884,1.040) \\
p=0.314\end{array}$ & & \\
\hline Technical.Control & & & & $\begin{array}{c}0.875 \\
(0.850,0.901) \\
\mathrm{p}<0.0001^{* *}\end{array}$ & \\
\hline DensityRural:Technical.Control & & & & $\begin{array}{c}0.998 \\
(0.917,1.086) \\
p=0.959\end{array}$ & \\
\hline Data.Transparency & & & & & $\begin{array}{c}0.977 \\
(0.952,1.002) \\
\mathrm{p}=0.075\end{array}$ \\
\hline DensityRural:Data.Transparency & & & & & $\begin{array}{c}0.983 \\
(0.912,1.060) \\
\mathrm{p}=0.656\end{array}$ \\
\hline Constant & $\begin{array}{c}0.005 \\
(0.004,0.005) \\
\mathrm{p}<0.0001^{* *}\end{array}$ & $\begin{array}{c}0.004 \\
(0.004,0.004) \\
\mathrm{p}<0.0001^{* *}\end{array}$ & $\begin{array}{c}0.004 \\
(0.004,0.004) \\
\mathrm{p}<0.0001^{* *}\end{array}$ & $\begin{array}{c}0.004 \\
(0.004,0.004) \\
\mathrm{p}<0.0001^{* *}\end{array}$ & $\begin{array}{c}0.004 \\
(0.004,0.004) \\
\mathrm{p}<0.0001^{* *}\end{array}$ \\
\hline $\begin{array}{l}\text { Observations } \\
\text { Log Likelihood } \\
\text { Akaike Inf. Crit. }\end{array}$ & $\begin{array}{c}6,858,820 \\
-178,438.100 \\
356,884.200\end{array}$ & $\begin{array}{c}6,858,820 \\
-178,732.500 \\
357,473.000\end{array}$ & $\begin{array}{c}6,858,820 \\
-178,707.500 \\
357,423.000\end{array}$ & $\begin{array}{c}6,858,820 \\
-178,688.200 \\
357,384.500\end{array}$ & $\begin{array}{c}6,858,820 \\
-178,733.900 \\
357,475.800\end{array}$ \\
\hline Note: & & & & & ${ }^{*} \mathrm{p}<0.05 ;{ }^{* *} \mathrm{p}<0.01$ \\
\hline
\end{tabular}

